\title{
Continuation phase cognitive therapy reduced relapse or recurrence in recurrent $D S M-I V$ major depressive disorder
}

\author{
Jarrett RB, Kraft D, Doyle J, et al. Preventing recurrent depression using cognitive therapy with and without a continuation \\ phase: a randomized clinical trial. Arch Gen Psychiatry 2001 Apr;58:381-8. \\ QUESTION: In patients with recurrent DSM-IV major depressive disorder (MDD) who \\ have responded to acute phase cognitive therapy, is continuation phase cognitive \\ therapy effective for reducing relapse or recurrence?
}

\section{Design}

Randomised (allocation concealed*), blinded (outcome assessors)*, controlled trial with 24 months of follow up.

\section{Setting}

University of Texas Southwestern Medical Center, Dallas, USA.

\section{Patients}

84 patients (mean age 43 y, 73\% women) with recurrent $D S M-I V$ MDD who responded to acute phase cognitive therapy and had a score of $\geqslant 16$ on the 17 item Hamilton Rating Scale for Depression at initial diagnostic evaluation and at follow up. Exclusion criteria were contraindicated medical condition or medication, exclusionary comorbid psychiatric disorder (MDD with psychotic features, current alcohol or other drug abuse), imminent suicide risk at triage, and inability to comply with protocol. $90 \%$ completed 8 months of the trial or had a relapse or recurrence.

\section{Intervention}

Patients entered 20 sessions of acute phase cognitive therapy (APCT). Unmedicated responders were allocated to either 10 sessions $(8 \mathrm{mo})$ of continuation phase cognitive therapy (CPCT) $(n=41)$ or control evaluation without cognitive therapy $(n=43)$. APCT focused on reducing symptoms and acquiring skills. The purpose of CPCT was to prevent relapse and recurrence, review strategies for symptom reduction, maintain skills acquired during APCT, and develop coping strategies in preparation for identified or anticipated vulnerabilities. Follow up lasted an additional 16 months after completion of treatment.

\section{Main outcome measures}

Relapse or recurrence of $D S M-I V \mathrm{MDD}$.

\section{Main results}

Analysis was by intention to treat. During the initial 8 months of follow up, the rate of relapse or recurrence in the CPCT group was less than the rate in the control group $(\mathrm{p}=0.02)$ (table).

\section{Conclusion}

In patients with recurrent $D S M-I V$ major depressive disorder who have responded to acute phase cognitive therapy, continuation phase cognitive therapy reduced relapse or recurrence rates when compared with the control (assessment without treatment).

*See glossary.
CPCT v control (assessment without treatment) for recurrent DSM-IV major depressive disorder after acute phase cognitive therapyt

\begin{tabular}{|c|c|c|c|c|}
\hline Outcome at 8 months & СРCT & Control & RRR $(95 \% \mathrm{CI})$ & NNT (Cl) \\
\hline Relapse or recurrence & $10 \%$ & $31 \%$ & $68 \%(10$ to 125$)$ & 5 (3 to 31$)$ \\
\hline
\end{tabular}

\section{COMMENTARY}

It is estimated that by 2006 the treatment of depression will be second only to ischaemic heart disease in the economic burden it places on healthcare services. ${ }^{1}$ The long term outcome in depression is disappointing, with estimates that $15-39 \%$ of cases will develop a chronic course. ${ }^{2}$ Thus the development of interventions to reduce the rate of relapse would not only alleviate suffering but ease economic burden. Cognitive therapy (CT) is as effective as antidepressant medication in the treatment of acute depression and evidence exists for its prophylactic effects in reducing relapse in chronic depression. ${ }^{3}$

What is original about the model proposed by Jarrett $e t$ al is that CT was delivered not only in the acute phase of illness but as a maintenance intervention after remission of the acute episode. The study is methodologically sound and the authors highlight its limitations and make recommendations for further research.

The trial generates evidence to support the prophylactic use of CT as a maintenance intervention, which may benefit patients for whom maintenance on medication is neither acceptable nor viable. What is advantageous is that the study used minimal exclusion criteria. Thus the sample is more likely to be representative of the heterogeneous presentations that characterise outpatients.

Importantly the authors have given consideration to the role of therapist competence in their analysis of patient outcome. Evidence is emerging that therapist competence and adherence to the CT treatment protocol are important in determining patient outcome. ${ }^{4}$ Thus in applying the results of efficacy studies in the clinical setting it is important to audit adherence to CT treatment protocols, that the protocols are delivered by clinicians trained in CT, and that CT supervision is an inherent component of service delivery.

Anne Garland, BA, RMN University of Glasgow, Gartnavel Royal Hospital Glasgow, UK

1 Jönsson B, Rosenbaum JF. Health economics of depression. Chichester; New York: Wiley, 1993.

2 Berti Ceroni G, Neri C, Pezzoli A. Chronicity in major depression: a naturalistic prospective study. J Affect Disord 1984;7:123-32.

3 Scott J. Treatment of chronic depression. N Engl J Med 2000;342:1518-20.

4 Scott J. Cognitive therapy for depression. Br Med Bull 2001;57:101-13.
Source of funding: National Institute of Mental Health.

For correspondence: Dr R B Jarrett, The University of Texas Southwestern Medical Center, Department of Psychiatry, 5323 Harry Hines Boulevard, Dallas, TX 75390-9149, USA $\mathrm{Fax}+1214648$ 5340 . website $e x t r a$

Additional information appears on the Evidence-Based Mental Health com 